OPEN ACCESS

Edited by:

Feng Liu,

Tianjin Medical University General

Hospital, China

Reviewed by:

Lei Gao,

Zhongnan Hospital of Wuhan

University, China

Ning Mao,

Peking University People's Hospital,

China

Heng Chen,

Guizhou University, China

${ }^{*}$ Correspondence:

Yunfei Zha

fskyanteam@126.com

Specialty section:

This article was submitted to Brain Imaging and Stimulation, a section of the journal

Frontiers in Human Neuroscience

Received: 01 June 2020

Accepted: 10 August 2020

Published: 04 September 2020

Citation:

Kuang C, Zha Y, Liu C and Chen J (2020) Altered Topological Properties

of Brain Structural Covariance Networks in Patients With Cervical

Spondylotic Myelopathy.

Front. Hum. Neurosci. 14:364. doi: 10.3389/fnhum.2020.00364

\section{Altered Topological Properties of Brain Structural Covariance Networks in Patients With Cervical Spondylotic Myelopathy}

\author{
Cuili Kuang, Yunfei Zha*, Changsheng Liu and Jun Chen \\ Department of Radiology, Renmin Hospital of Wuhan University, Wuhan, China
}

Background: Brain structural alterations play an important role in patients with cervical spondylotic myelopathy (CSM). However, while there have been studies on regional brain structural alterations, only few studies have focused on the topological organization of the brain structural covariance network. This work aimed to describe the structural covariance network architecture alterations that are possibly linked to cortex reorganization in patients with CSM.

Methods: High-resolution anatomical images of 31 CSM patients and 31 healthy controls ( $\mathrm{HCs}$ ) were included in the study. The images were acquired using a sagittal three-dimensional T1-weighted BRAVO sequence. Firstly, the gray matter volume of 90 brain regions of automated anatomical labeling atlas were computed using a VBM toolbox based on the DARTEL algorithm. Then, the brain structural covariance network was constructed by thresholding the gray matter volume correlation matrices. Subsequently, the network measures and nodal property were calculated based on graph theory. Finally, the differences in the network metrics and nodal property between groups were compared using a non-parametric test.

Results: Patients with CSM showed larger global efficiency and smaller local efficiency, clustering coefficient, characteristic path length, and sigma values than HCs. Patients with CSM had greater betweenness in the left superior parietal gyrus (SPG.L) and the left supplementary motor area (SMA.L) than HCs. Besides, patients with CSM had smaller betweenness in right middle occipital gyrus. The brain structural covariance networks of CSM patients exhibited equal resilience to random failure as those of HCs. However, the maximum relative size of giant connected components was approximately $10 \%$ larger in HCs than in CSM patients, upon removal of 44 nodes in targeted attack.

Conclusion: These observed alternations in global network measures in CSM patients reflect that the brain structural covariance network in CSM exhibits the less optimal small-world model compared to that in HCs. Increased betweenness in SPG.L and 
SMA.L seems to be related to cortex reorganization to recover multiple sensory functions after spinal cord injury in CSM patients. The network resilience of patients with CSM exhibiting a relative mild vulnerability, compared to HCs, is probably attributable to the balance and interplay between cortex reorganization and ongoing degeneration.

\section{Keywords: cervical spondylotic myelopathy, brain structural covariance network, graph theory analysis, small} world, topological properties

\section{INTRODUCTION}

Cervical spondylotic myelopathy (CSM) is a common spinal cord dysfunction that causes motor and sensory deficits of the limbs, neck pain, and cervical vertigo with gait instability (Yarbrough et al., 2012). Usually, CSM is regarded as a specific form of incomplete spinal cord injury (SCI) (Sharp and Simon, 2005). Many brain studies have reported functional and structural changes in the cortex of patients with CSM, and brain reorganization after SCI has been accepted as the pivotal factor affecting the function and rehabilitation of patients with CSM (Zhou et al., 2014; Chen et al., 2019). Cortical damage or plasticity in the central nervous system has also been suggested to influence the clinical symptoms, manifestations, and functional rehabilitation of patients with CSM (Zhou et al., 2014). Specifically, functional magnetic resonance imaging (MRI) studies have reported increased volume of activation within the primary motor cortex and decreased volume of activation within the primary sensory cortex in patients with CSM, followed by cortical reorganization after decompressive surgery (Dong et al., 2008; Holly, 2009; Duggal et al., 2010). A magnetic resonance spectroscopy study detected decreased $\mathrm{N}$-acetylaspartate/creatine, which implied axonal or neuronal damage/loss in the motor cortex of patients with CSM, and the presence of neurological damage was suggested to be linked to axonal and/or neuronal injury (Kowalczyk et al., 2012). Furthermore, a recent study has reported decreased gray matter volume (GMV) in the left superior parietal lobule and decreased white matter (WM) volume in the right temporal lobe, occipital lobule, and calcarine gyrus in patients with subacute incomplete cervical cord injury (Chen et al., 2019). Alterations in the central nervous system not only occur in incomplete SCI, other brain studies focusing on patients with complete SCI have also observed gray matter alterations. A previous voxel-based morphometry (VBM) study detected significant gray matter atrophy in the primary motor cortex, primary somatosensory cortex, supplementary motor area, and thalamus during the early stage of SCI in humans (Hou et al., 2014). Another

\footnotetext{
Abbreviations: AAL, automatic anatomic labeling; AUC, areas under curve; $\mathrm{Cp}$, clustering coefficient; CSM, cervical spondylotic myelopathy; DARTEL, diffeomorphic anatomical registration through exponentiated lie algebra; fMRI, functional magnetic resonance imaging; GAT, graph analysis toolbox; GM, gray matter; GMV, gray matter volume; HCs, healthy controls; JOA, Japanese Orthopaedic Association; Lp, characteristic path length; MOG.R, right middle occipital gyrus; MOG, middle occipital gyrus; MRI, magnetic resonance imaging; NDI, neck disability index; SCI, spinal cord injury; SD, standard deviation; SMA.L, left supplementary motor area; SMA, supplementary motor area; SPG.L, left superior parietal gyrus; SPG, superior parietal gyrus; SPM8, statistical parametric mapping; VBM, voxel-based morphometry; WM, white matter.
}

study found decreased GMV in the anterior cingulate cortex, left insula, left secondary somatosensory cortex, and bilateral thalamus in patients with chronic traumatic SCI (Jutzeler et al., 2016). Importantly, a previous study reported that progressive atrophic and microstructural changes across the sensory system were closely related to sensory outcome in patients with SCI (Grabher et al., 2015). These findings suggest that structural alterations play a key role in patients with incomplete or complete SCI. However, while these studies have provided important information on regional brain structural alterations, only few have focused on the topological organization of the brain structural covariance network.

Nowadays, brain structural covariance network analysis relying on morphological metrics such as the GMV and cortical thickness is widely applied in neuropathological mechanism researches (He et al., 2007; Wen et al., 2011). Based on the statistical correlations of the morphological descriptors and the graph theory method, network analysis can explore the topological organization of the whole brain, thus providing comprehensive network-level information (He et al., 2007; Lv et al., 2010). This type of information extends and complements conventional brain structural MRI findings. The biological basis of structural covariance network analyses is the interregional correlations of gray matter volumes or thicknesses, which are considered to represent brain regional connectivity of the network (Sone et al., 2019). This method can extract measures of inter-regional connectivity from the covariance patterns of gray matter volumes or thicknesses and may provide more knowledge on neuropsychiatric diseases when used in combination with functional or diffusion imaging (AlexanderBloch et al., 2013; Evans, 2013; Sone et al., 2019). In largescale complex human brain network analysis, this type of brain structural covariance network possesses small-world properties and the other topological properties, such as network centrality and resilience. A small-world network with extensive local clustering and short path lengths makes it an attractive model for information processing through minimal wiring costs (Sporns et al., 2005; Achard and Bullmore, 2007). In other words, a small-world network processes high global efficiency reflecting the network's capacity for parallel information transfer and high local efficiency indicating the information transfer between adjacent nodes. The nodal betweenness is commonly considered as the nodal centrality and quantifies the influence of a node in connecting other nodes in a network (Freeman, 1977; Cheng et al., 2015). A node with high betweenness is thus crucial to efficient communication and can reach the other nodes on short paths (Barthélemy, 2004). The betweenness equals the fraction of all shortest paths in the network that pass through a given 
node (Rubinov and Sporns, 2010; Cheng et al., 2015) and reflects the dynamic performances of the network, such as the speed of information transfer ( $\mathrm{He}$ et al., 2008). Resilience indicates the tolerance of the network against random or targeted attack, and is relevant to the stability of the network (Achard et al., 2006; He et al., 2008). Various neurological diseases are characterized by the topological properties of the brain structural covariance network (Yao et al., 2010; Shi et al., 2012; Tao et al., 2018; Zou et al., 2018; Liu et al., 2019), such as the small-world property, network centrality, and resilience (He et al., 2008, 2009).

In this study, prior to constructing a brain structural covariance network, we used a VBM toolbox based on the diffeomorphic anatomical registration through exponentiated lie algebra (DARTEL) algorithm to obtain the precise GMV as the network morphological descriptor (Ashburner, 2007) and constructed the brain structure covariance network using the graph analysis toolbox (GAT) (Hosseini et al., 2012). Next, we calculated and compared global and local efficiency, smallworld parameters, nodal betweenness, and resilience of the brain structural covariance networks of the patients with CSM and healthy controls (HCs). We hypothesized that the changes in the patterns of the network properties evaluated in the patients with CSM will correspond to the patterns of cortex reorganization, which has been reported in previous studies, and we expected the existence of a potential link between the alternation of network properties and cortex organization in patients with CSM.

\section{MATERIALS AND METHODS}

\section{Participants}

A total of 33 right-handed patients with CSM [17 women and 16 men; age range, 42-67 years; mean age \pm standard deviation (SD), $54.78 \pm 8.41$ years] were recruited at the Renmin Hospital of Wuhan University through convenience sampling. The mean duration of symptoms from disease onset to the date of MRI examination was $37.0 \pm 25.1$ months (range, 3 months to 8 years). All the patients met the following inclusion criteria: (1) cervical spondylosis, (2) an ossified posterior longitudinal ligament, and (3) demyelination with hyperintensity of cord on T2-weighted imaging (Zhou et al., 2014). Two radiologists determined the spinal cord compression level at which the cord surface was clearly indented, or cord diameter was narrowed by compression. The exclusion criteria were as follows: trauma- or infectionrelated cord compression, any other neurological disorder, a history of decompression surgery, and any contraindications for MRI. The clinical severity of myelopathy was assessed using the Japanese Orthopaedic Association (JOA) score system (Yonenobu et al., 2001) and the neck disability index (NDI) questionnaire. The JOA score system evaluates the severity of myelopathy by assigning scores based on the degree of dysfunction, and the NDI questionnaire measures the activities of daily living in patients with neck pain. As HCs, 33 right-handed age- and sex-matched healthy volunteers (18 women and 15 men; age range, $40-63$ years; mean age $\pm S D, 53.52 \pm 8.13$ years) were recruited via community health screenings. Two patients with CSM and two HCs were excluded due to obvious blur artifacts observed in the structural images. Finally, 31 patients with CSM and $31 \mathrm{HCs}$ were included in the analyses. The current study was approved by the ethics committee for clinical research of Renmin Hospital of Wuhan University and was conducted in accordance with the principles of the Declaration of Helsinki. All the participants gave written informed consent.

\section{MRI Procedure}

A GE Discovery 750 3.0-Tesla MR scanner equipped with an eight-channel head coil was used to acquire the MR images. High-resolution anatomical images were acquired using a sagittal three-dimensional T1-weighted BRAVO sequence with the following parameters: repetition time/echo time $=7.2 / 2.7 \mathrm{~ms}$, inversion time $=450 \mathrm{~ms}$, flip angle $=12^{\circ}$, number of slices $=160$, slice thickness $=1.0 \mathrm{~mm}$, field of view $=25.6 \mathrm{~cm} \times 25.6 \mathrm{~cm}$, readout bandwidth $=41.67 \mathrm{kHz}$, and in-plane matrix $=256 \times 256$. Sagittal and axial conventional T1-weighted, T2-weighted, and T2 fluid-attenuated inversion recovery images of the brain and cervical spinal cord were acquired for patient diagnosis. For the two groups, the time period of data acquisition was from March 2017 to May 2018.

\section{Data Preprocessing Measurements of the GMV}

To obtain the GMV, we used a VBM8 toolbox based on the DARTEL algorithm (Ashburner, 2007). VBM8 was performed using statistical parametric mapping $(\mathrm{SPM} 8)^{1}$ running on MATLAB (R2013a, Mathworks, Natick, MA, United States). First, each subject's structural MR images were segmented into gray matter (GM), WM, and cerebrospinal fluid in the nativespace using the segmentation module in SPM8 (Ashburner and Friston, 2005). Secondly, the study-specific DARTEL templates (GM, WM) were generated from the entire image dataset using the DARTEL technique (Ashburner, 2007). Thirdly, the DARTEL templates were affine registered to the tissue probability maps in the Montreal Neurological Institute (MNI) space. Then non-linear warping of the native-space segmented images was normalized to match the DARTEL templates (GM, WM) in the MNI space, during this procedure, images were modulated to ensure that relative volumes of GM and WM were preserved following the spatial normalization. Finally, the modulated and normalized images were smoothed with a 6-mm full-width at half-maximum isotropic gaussian kernel.

\section{Construction of the Brain Structural Covariance Network}

Pearson correlation coefficients between smoothed, modulated, and normalized GMVs were calculated across all participants for each group to generate $\mathrm{N} \times \mathrm{N}$ interregional correlation matrices using the GAT (Hosseini et al., 2012; Figures 1A,C; binarized, Figures 1B,D) (N represents the number of nodes). We selected 90 regions of interest of the automatic anatomic labeling (AAL) atlas as nodes (Tzourio-Mazoyer et al., 2002). A linear regression analysis was performed to remove the influence of nuisance covariates, namely age, sex, and total intracranial volume, in

${ }^{1}$ http://www.fil.ion.ucl.ac.uk/spm 
A

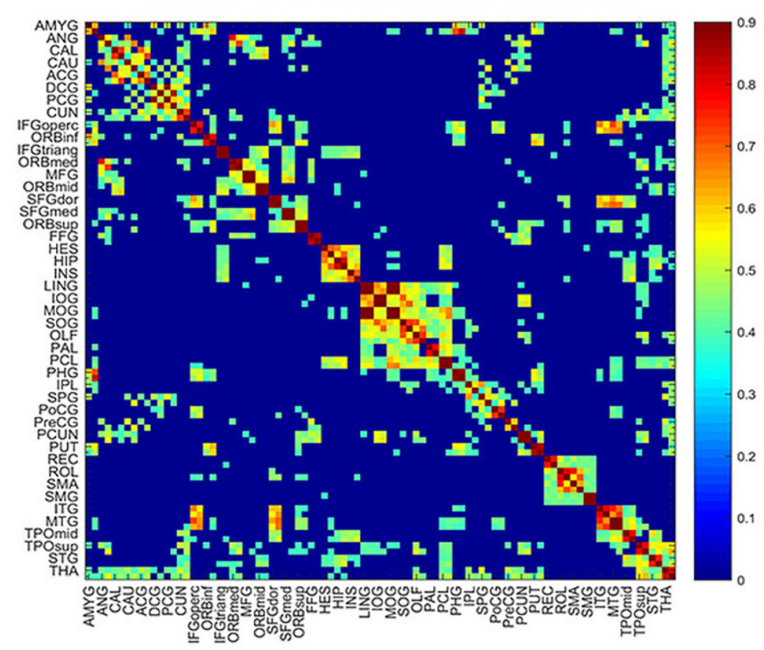

C

c CSM Covariance Matrix

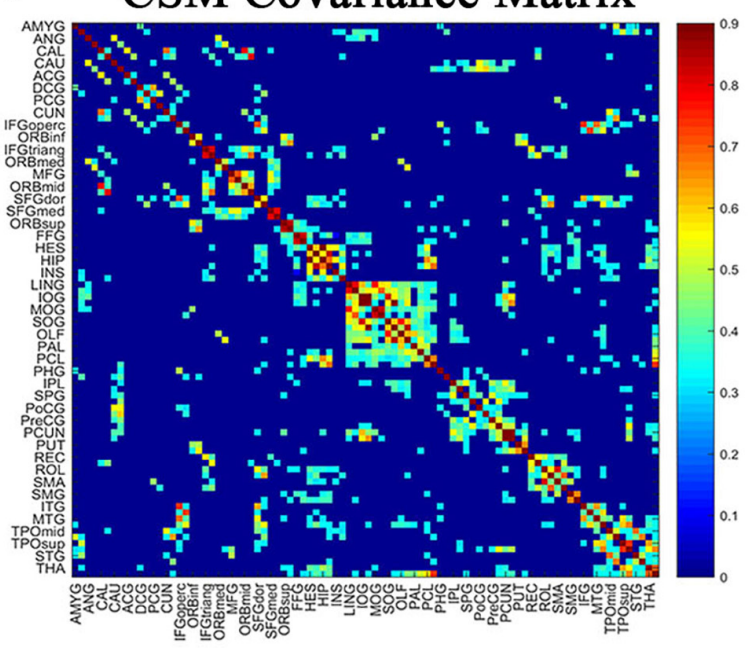

B

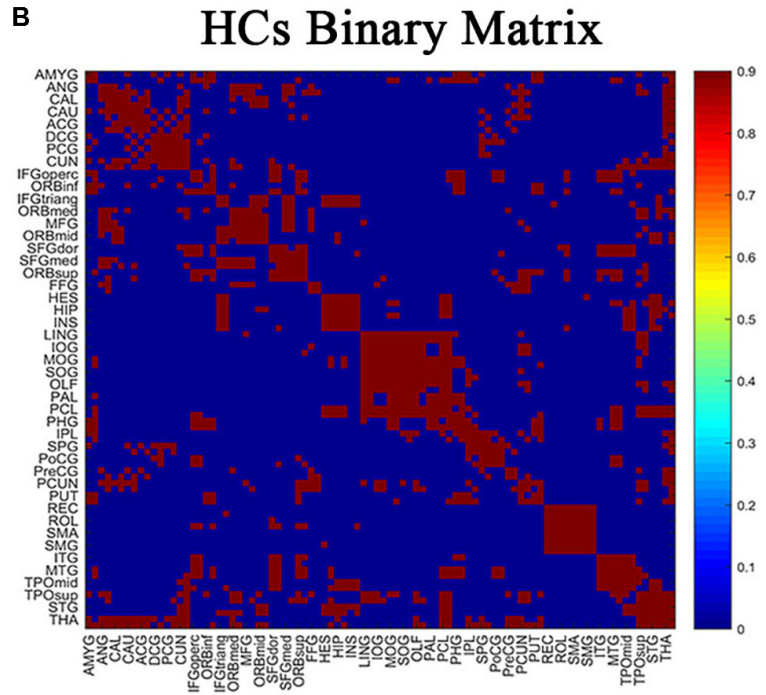

D

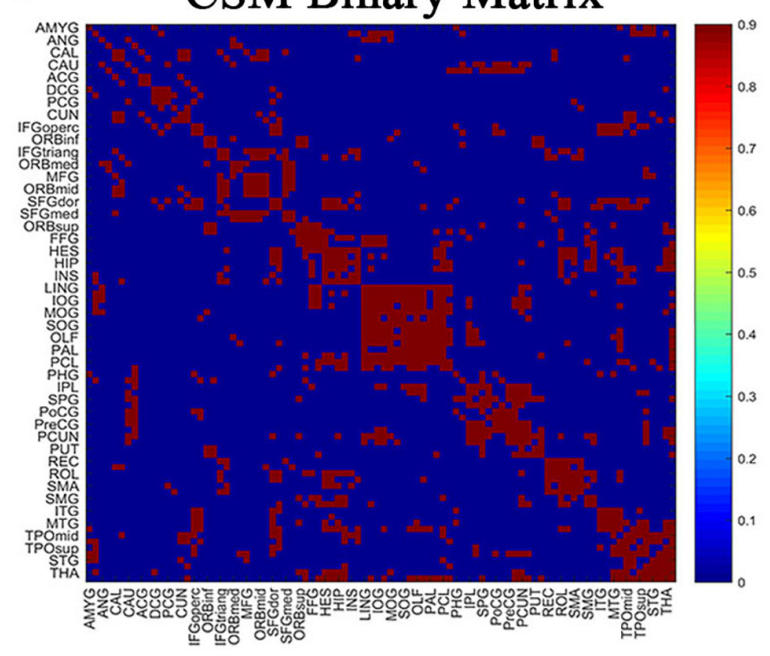

FIGURE 1 | The correlation matrices of healthy controls (HCs) $\mathbf{( A , B )}$ and patients with cervical spondylotic myelopathy (CSM) groups (C,D) (left, thresholded; right, binarized). The color bar indicates the strength of the Pearson correlation coefficients between brain areas. The $X / Y$ axes represent the $90 \mathrm{ROIs}$ from the AAL atlas, with the exception of the 26 cerebellar regions. For clarity, brain regions of AAL atlas without distinction between left and right are labeled. For the full forms of the abbreviations of the brain regions, see Table 1.

each group. Then, the interregional correlation matrices were thresholded across a range of network densities ( 0.1 to 0.5 with an interval of 0.02). The minimum density guaranteed that the network for each of the two groups was fully connected. The maximum density prevented the networks from exhibiting randomness. Previous studies have indicated that a maximum network density should be below 50\% since structural networks with more than $50 \%$ connections are likely non-biological (Kaiser and Hilgetag, 2006; Hosseini et al., 2012). Lastly, network topologies were calculated and compared between the two groups at each density.

\section{Graph Theory Analysis}

Clustering coefficient (Cp) and characteristic path length (Lp) are two crucial metrics of the complex network
(Bullmore and Sporn, 2009; Rubinov and Sporns, 2010). Cp is the average clustering coefficient across all nodes in a network and estimates the functional segregation for specialized processing to occur within densely interconnected groups of brain regions (Watts and Strogatz, 1998; Rubinov and Sporns, 2010). Lp is the average shortest path length between all pairs of nodes in the network and is the most commonly used measure of functional integration between brain regions (Watts and Strogatz, 1998; Rubinov and Sporns, 2010). To characterize the topological properties of the brain network, the two metrics are compared to the null random networks (20 generated null networks). Thereafter, the normalized $\mathrm{Cp}\left(\gamma=\mathrm{Cp} / \mathrm{Cp}_{\text {rand }}\right)$, the normalized $\operatorname{Lp}\left(\lambda=\mathrm{Lp} / \mathrm{Lp}_{\text {rand }}\right)$, and the small-world index Sigma $(\sigma=\gamma / \lambda)$ can be obtained. If $\gamma>1$, and $\lambda=\sim 1$ or $\sigma>1$, the brain network possesses small-world properties. This implies 
TABLE 1 | Abbreviations of brain regions of AAL atlas (without distinction between left and right).

\begin{tabular}{|c|c|c|c|}
\hline Abbreviation & AAL region & Abbreviations & AAL regions \\
\hline AMYG & Amygdala & MOG & Middle occipital gyrus \\
\hline ANG & Angular gyrus & SOG & Superior occipital gyrus \\
\hline $\mathrm{CAL}$ & Calcarine cortex & OLF & Olfactory cortex \\
\hline CAU & Caudate nucleus & PAL & Lenticular nucleus, pallidum \\
\hline ACG & Anterior cingulate gyrus & PCL & Paracentral lobule \\
\hline DCG & Middle cingulate gyrus & PHG & Parahippocampal gyrus \\
\hline PCG & Posterior cingulate gyrus & IPL & Inferior parietal lobule \\
\hline CUN & Cuneus & SPG & Superior parietal gyrus \\
\hline IFGoperc & Inferior frontal gyrus (opercular) & PoCG & Postcentral gyrus \\
\hline ORBinf & Inferior frontal gyrus (inferior) & PreCG & Precentral gyrus \\
\hline IFGtriang & Inferior frontal gyrus (triangular) & PCUN & Precuneus \\
\hline ORBmed & Orbitofrontal cortex (medial) & PUT & Lenticular nucleus, putamen \\
\hline MFG & Middle frontal gyrus & REC & Rectus gyrus \\
\hline ORBmid & Orbitofrontal cortex (middle) & ROL & Rolandic operculum \\
\hline SFGdor & Superior frontal gyrus (dorsal) & SMA & Supplementary motor area \\
\hline SFGmed & Superior frontal gyrus (medial) & SMG & Supramarginal gyrus \\
\hline ORBsup & Orbitofrontal cortex (superior) & ITG & Inferior temporal gyrus \\
\hline FFG & Fusiform gyrus & MTG & Middle temporal gyrus \\
\hline HES & Heschl gyrus & TPOmid & Temporal pole (middle) gyrus \\
\hline HIP & Hippocampus & TPOsup & Temporal pole (superior) gyrus \\
\hline INS & Insula & STG & Superior temporal gyrus \\
\hline LING & Lingual gyrus & THA & Thalamus \\
\hline IOG & Inferior occipital gyrus & & \\
\hline
\end{tabular}

that the clustering coefficient of the brain structural network is significantly higher than that of random networks, and the characteristic path length is comparable to that of random networks (Bullmore and Sporn, 2009; Rubinov and Sporns, 2010). Global efficiency is inversely related to average minimum path length and measures the network's capacity for parallel information transfer between nodes via multiple series of edges (Achard and Bullmore, 2007). Local efficiency measures the information transfer in the immediate neighborhoods of each node and indicates the fault tolerance of the network to deletion of individual nodes (Achard and Bullmore, 2007).

For regional nodal characteristics of the brain structural covariance network, we discussed and measured the normalized nodal betweenness (nodal betweenness normalized by the average betweenness of the network). The normalized nodal betweenness captures the influence of a node over information flow between other nodes in the network (He et al., 2008). The network metrics, small-world parameter, and normalized nodal betweenness were calculated using the GAT.

In previous studies, resilience have been investigated in brain networks (Achard et al., 2006; Kaiser et al., 2007). Resilience indicates the tolerance of the brain network to random failure or targeted attack. Random failure could be simulated by removing one node from the network and targeted attack could be evaluated by removing nodes in the order of decreasing degree or betweenness. These processes can be repeated until all of the nodes are removed. The resilience can be visualized by plotting the size of the giant connected components as a function of the number of nodes removed (Achard et al., 2006). The relative size of the giant connected component represents the size of the giant connected component normalized by the biggest connected component of the network before the nodes are removed. In the present study, we first randomly removed a node from the brain networks of the two groups and measured the relative size of the giant connected component. We repeated the process until all the nodes were removed. Subsequently, we removed nodes in order of decreasing betweenness. Similarly, the process was repeated until all the nodes were removed. Lastly, we obtained the curves describing the changes in the relative size of the giant connected component as a function of the number of nodes removed.

\section{Statistical Analyses}

The distribution of age and sex between groups was assessed using the two-sample $t$-test and the chi-squared test, respectively. The differences in network measures between the two groups were tested using a non-parametric permutation algorithm (Bullmore et al., 1999; He et al., 2008; Liu et al., 2016; Zou et al., 2018). First, we calculated the network metrics (Cp, Lp), small-world parameter (Sigma), and nodal characteristic (nodal betweenness) separately for the two groups across the density range (0.1:0.02:0.5). Next, we randomly rearranged each participant's set of modulated GMV data to one or the other of the two groups and computed the correlation matrix for each randomized group, after which we calculated the network metrics and nodal characteristic for each randomized group across the same density range (0.1:0.02:0.5) as in the real brain networks. The randomization procedure was repeated 1000 times. Lastly, we employed the 95 percentile points of each distribution as the 
critical values for a one-tailed test of the null hypothesis with a type I error probability of 0.05 (He et al., 2008) and extracted the curves that presented the difference in network metrics (between two groups) as functions of network density.

Subsequently, in order to reduce the impact of thresholding process, we compared the curve differences between groups through a summary measure using areas under a curve (AUC) analysis. To test the significance of the between-group differences in AUC of each network measure, a similar approach of a nonparametric permutation algorithm was applied (Hosseini et al., 2012). First, the real between-group difference in AUC for each network measure was calculated. Then, for each randomized group, the between-group difference in AUC for each network measure was computed for the 1000 times of randomization procedure. Lastly, the 95 percentile points of each distribution as the critical values for a one-tailed test of the null hypothesis with a type I error probability of 0.05 was used.

To test whether the two group networks behaved differently against random failure and targeted attack, we applied a permutation analysis following the procedure mentioned for analyzing between-group differences in network measures, which has been performed in a previous study (Hosseini et al., 2012). First, the real between-group difference in network resilience against random failure and targeted attack by the deletion of nodes were calculated. Next, for each randomized group, the between-group difference in network resilience against random failure and targeted attack by the deletion of nodes (as calculated in the real brain networks) were computed. Lastly, the 95 percentile points of each distribution as the critical values for a one-tailed test of the null hypothesis with a type I error probability of 0.05 was used.

\section{RESULTS}

\section{Demographic and Clinical Data Profiling}

Table 2 summarizes the demographic and clinical data of patients with CSM and HCs. There were no significant differences in age $(P=0.752)$ and sex $(P=0.95)$ between the two groups.

TABLE 2 | Demographic and clinical data of patients with CSM and HCs.

\begin{tabular}{lccc}
\hline Subject & CSM group & HCs group & $\boldsymbol{P}$-value \\
\hline $\mathrm{N}$ & 31 & 31 & $\mathrm{n} / \mathrm{a}$ \\
$\mathrm{Age}$ & $54.78 \pm 8.41$ & $53.52 \pm 8.13$ & 0.752 \\
Gender (male/female) & $16 / 17$ & $15 / 18$ & 0.95 \\
Handedness (right/left) & $31 / 0$ & $31 / 0$ & $\mathrm{n} / \mathrm{a}$ \\
Laterality of spinal cord & $17 / 12 / 4$ & $\mathrm{n} / \mathrm{a}$ & $\mathrm{n} / \mathrm{a}$ \\
compression & & & \\
(right/left/bilateral) & & $17 \pm 0$ & $<0.0001$ \\
JOA scores & $10.53 \pm 2.57$ & $0.009 \pm 0.001$ & $<0.0001$ \\
NDI scores & $0.345 \pm 0.102$ & $\mathrm{n} / \mathrm{a}$ & $\mathrm{n} / \mathrm{a}$ \\
$\begin{array}{l}\text { Duration of symptoms } \\
\text { (month) }\end{array}$ & $37.0 \pm 25.1$ & & \\
\hline
\end{tabular}

CSM, cervical spondylotic myelopathy; HCs, healthy controls; JOA, Japanese Orthopaedic Association; NDI, neck disability index.
However, JOA scores, NDI scores, and duration of symptoms were significantly different between the two groups $(P<0.0001)$.

\section{Network Analyses \\ Global Network Measures and Small-World Parameter}

In the current study, we investigated the between-group differences in global network measures and small-world parameter on brain structural covariance network at a range of densities (0.1:0.02:0.5). Compared with HCs, the network of patients with CSM showed greater global efficiency (Figure 2A) and smaller local efficiency (Figure 2B), Lp (Figure 2C), Cp (Figure 2D), and Sigma (Figure 2E) at several densities across the range $(P<0.05)$. In addition to comparing the networks at various densities, we compared the AUC for global network measures and small-world parameter curves (across the density range of 0.1:0.02:0.5) between the two groups. We observed that the network of patients with CSM had significantly larger AUC for global efficiency $(P=0.05)$ and smaller AUC for local efficiency $(P=0.04), \mathrm{Lp}(P=0.05), \mathrm{Cp}(P=0.02)$, and Sigma $(P=0.04)$ (Figure 2F).

\section{Nodal Betweenness}

Figure 3 shows the differences (azure dots) in nodal betweenness in 45 left cerebral regions (Figure 3A) and 45 right cerebral regions (Figure $3 \mathbf{B}$ ) of AAL atlas between patients with CSM and HCs. Table 3 lists the brain regions with significant changes in nodal betweenness in patients with CSM compared with that in HCs. Figure 4 highlights these brain regions in anatomical space. The red (blue) nodes indicate increased (decreased) nodal betweenness in the CSM group compared to that in the HCs group.

\section{Network Resilience}

Figure 5A shows that the brain structural covariance network of the CSM group was as resilient to random failure as that of the HCs group. However, in targeted attack, when the number of nodes removed were less than 37 (Figure 5B, dotted line indicated with (1) or more than 63 (Figure 5B, dotted line indicated with (3), the relative size of giant connected components of the two groups were equivalent. When the number of nodes removed were in the range of 37 to 63 , the maximum difference of the relative size of giant connected components between the two groups was nearly 10\% when 44 nodes were removed (Figure 5B, dotted line indicated with (2)). The difference in the relative sizes of giant connected components between the two groups was significant when 44, 45, and 50 nodes were removed (where the red stars indicate in Figure 5B).

\section{DISCUSSION}

Here, we used graph theory-based network analyses to compare the topological properties of the brain structural covariance networks between patients with CSM and HCs. We found that patients with CSM had larger global efficiency and smaller local efficiency, Lp, Cp, and Sigma values than HCs. Patients with 

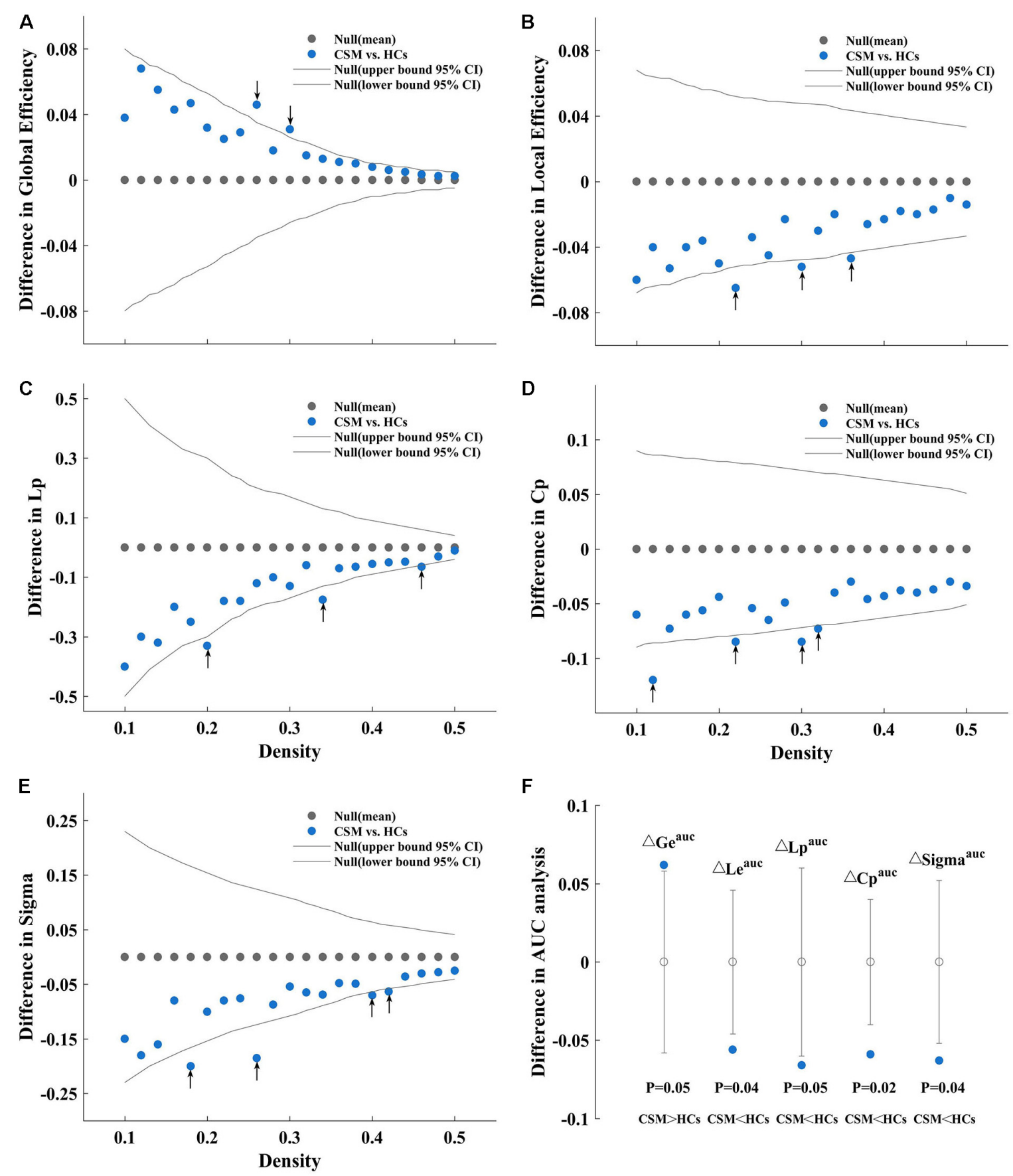

$-0.1^{L}$

CSM $>$ HCs CSM $<$ HCs CSM $<$ HCs $\mathrm{CSM}<\mathrm{HCs}$ CSM $<$ HCs

FIGURE 2 | Between-group differences in global efficiency (A), local efficiency (B), characteristic path length (Lp) (C), clustering coefficient (Cp) (D), and Sigma (E) as a function of density. Between-group differences in areas under the global efficiency ( $\left.\Delta G e^{\text {auc }}\right)$, local efficiency ( $\left.\Delta \mathrm{Le}^{\text {auc }}\right), \operatorname{Lp}\left(\Delta \mathrm{Cp}{ }^{\text {auc }}\right), \mathrm{Cp}\left(\Delta \mathrm{Lp} p^{\text {auc }}\right)$, and $\operatorname{Sigma}$ $\left(\Delta\right.$ Sigma auc $^{\text {aurves }} \mathbf{( F )}$ ), from left to right sequentially). As an example, we have described (A). (A) Denotes the differences (azure dots) in the global efficiency between the patients with cervical spondylotic myelopathy (CSM) and the healthy controls (HCs) as a function of density thresholds. The gray dots represent the mean values and the gray lines represent the $95 \%$ confidence intervals of the between-group differences obtained 1000 permutation tests at each density value. The black arrows indicate significant difference in global efficiency between the two groups $(P<0.05)$. Patients with CSM show larger global efficiency in the brain structural covariance networks than HCs at thresholds 0.26 and 0.30. Similarly, (B) denotes that patients with CSM show smaller local efficiency in the brain structural covariance networks than HCs at threshold 0.22, 0.30 , and 0.36. (C) Denotes that patients with CSM show smaller Lp in the brain structural covariance networks than $\mathrm{HCs}$ at threshold $0.20,0.34$, and 0.46. (D) Denotes that patients with CSM show smaller Cp in the brain structural covariance networks than HCs at threshold 0.12, 0.22, 0.30, and 0.32. (E) Denotes that patients with CSM show smaller Sigma in the brain structural covariance networks than HCs at thresholds $0.18,0.26,0.40$, and 0.42. (F) Denotes that patients with CSM show significantly larger Ge $\mathrm{C}^{\text {auc }}(P=0.05)$ and smaller $\mathrm{Le}^{\text {auc }}(P=0.04), \mathrm{Lp}^{\text {auc }}(P=0.05), \mathrm{Cp} p^{\text {auc }}$ $(P=0.02)$, and Sigma auc $(P=0.04)$ in the brain structural covariance networks than HCs in the areas under curve (AUC) analysis. 


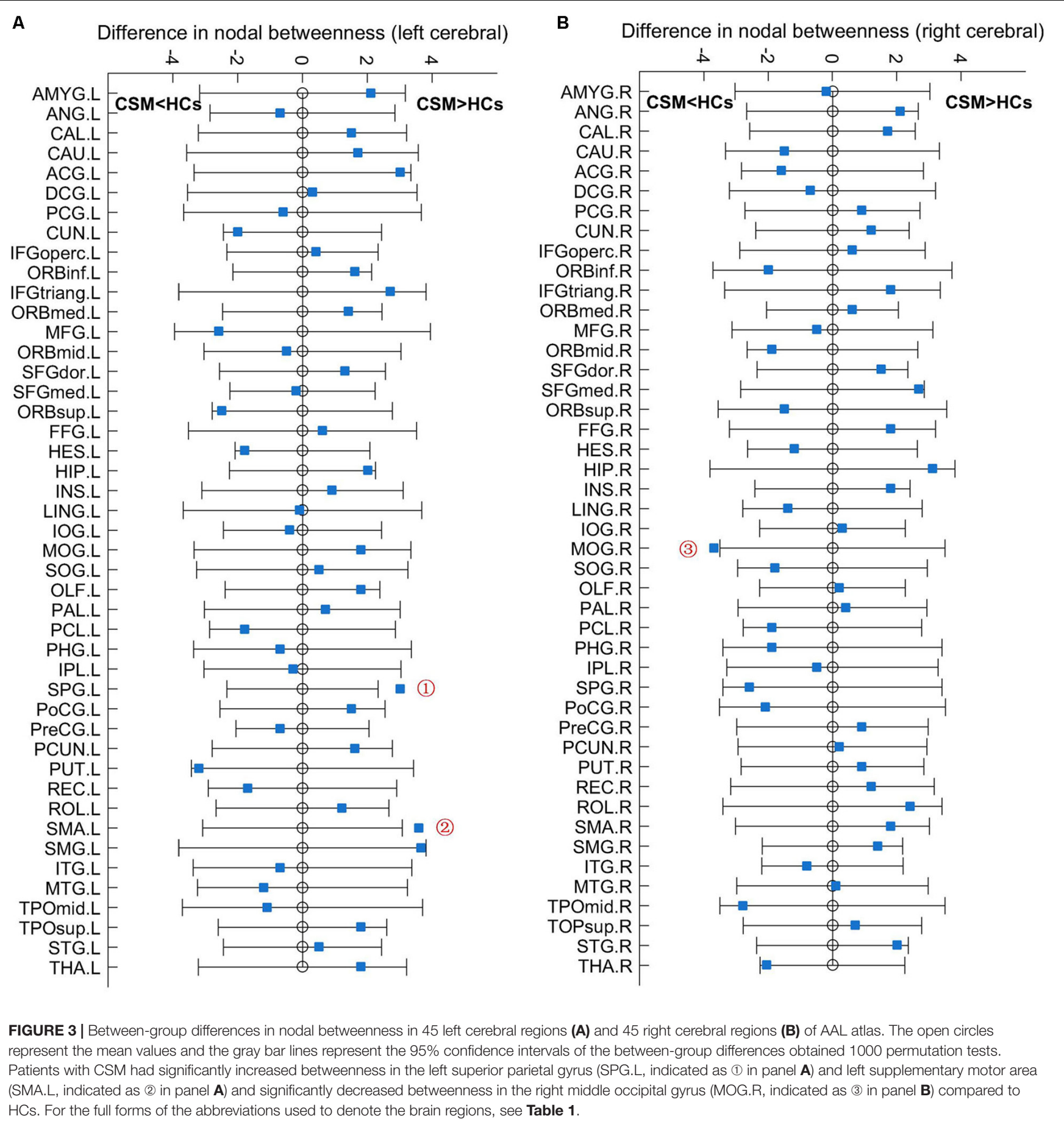

TABLE 3 | AUC analysis for nodal betweenness in patients with CSM and HCs.

\begin{tabular}{|c|c|c|c|c|}
\hline Relationship & AAL region & CSM betweenness & HCs betweenness & $P$-value \\
\hline \multirow[t]{2}{*}{$\mathrm{CSM}>\mathrm{HCS}$} & Left superior parietal gyrus & 3.63 & 0.28 & 0.01 \\
\hline & Left supplementary motor area & 2.59 & 0.46 & 0.03 \\
\hline $\mathrm{CSM}<\mathrm{HCS}$ & Right middle occipital gyrus & 0.07 & 0.52 & 0.05 \\
\hline
\end{tabular}

CSM, cervical spondylotic myelopathy; HCs, healthy controls; AUC, areas under the curve; AAL, automatic anatomic labeling. 

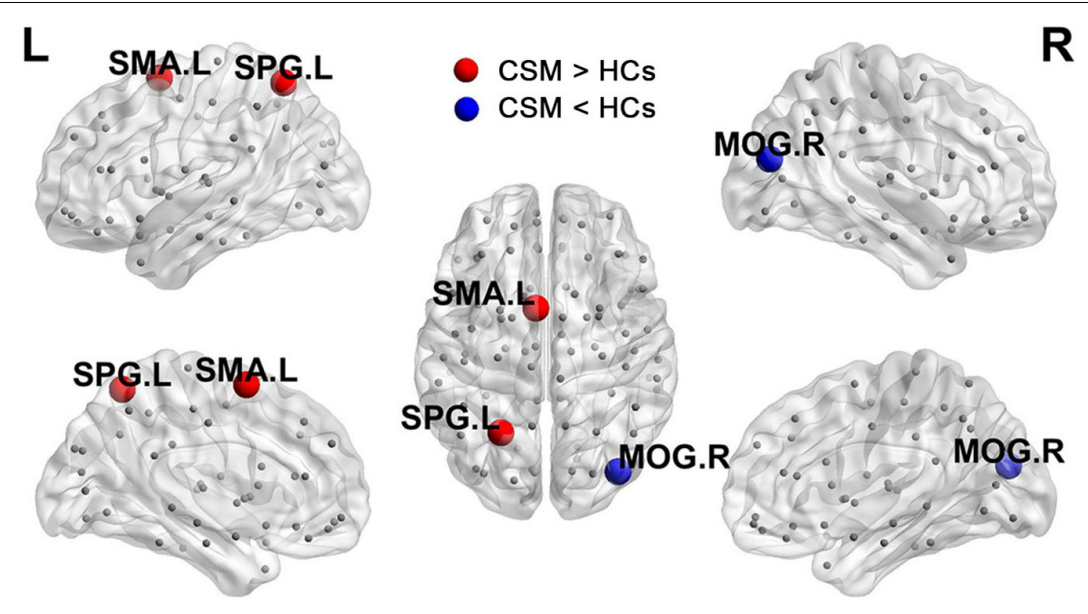

$\mathbf{R}$

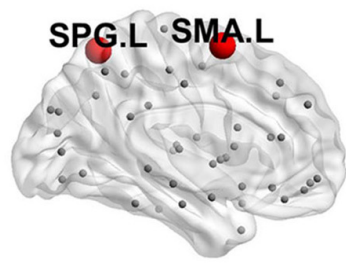

FIGURE 4 | Brain regions with significant changes in nodal betweenness in patients with CSM compared to HCs.
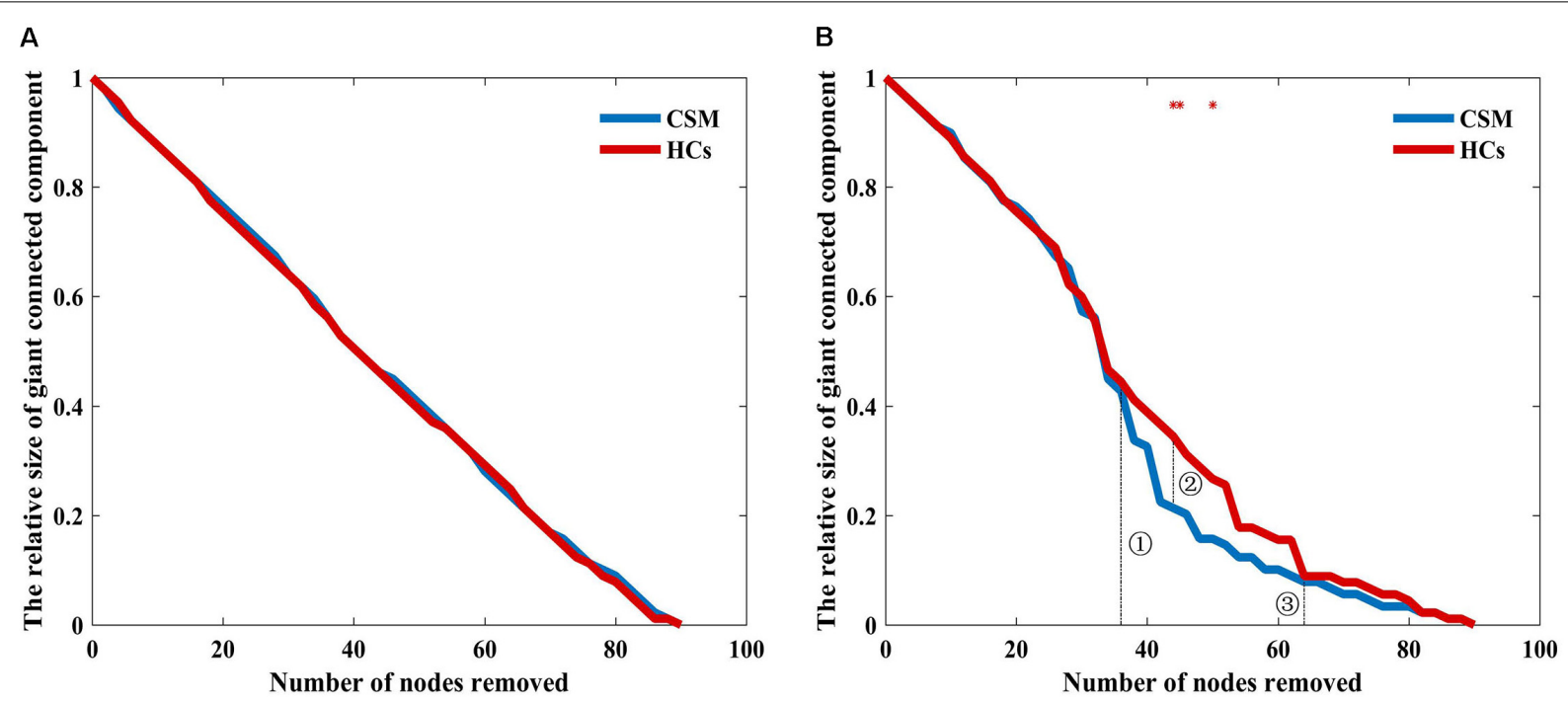

FIGURE $\mathbf{5}$ | Relative size of the giant connected component as a function of the number of nodes removed by random failure (A) and targeted attack (B) in the two groups. Red stars show where the difference in the relative size of the giant connected component between the groups is statistical significant $(P<0.05)$.

CSM had increased nodal betweenness in the SMA.L, SPG.L, and decreased betweenness in the MOG.R compared to that in the HCs. Both groups exhibited equal resilience to random failure. When 44 nodes were removed in the analysis for targeted attack, the maximum relative size of giant connected components was approximately $10 \%$ larger in HCs than in patients with CSM. Further, the difference in the relative size of giant connected components between the two groups was significant upon removal of several different number of nodes.

\section{Altered Global Network Measures and Small-World Parameter in Patients With CSM}

The small-world network reflects an attractive model for the combination of high clustering and short path length, which confers a capability for both specialized or modular processing in local neighborhoods and distributed or integrated processing over the entire network (Achard et al., 2006). In the present study, the network measures Cp, Lp, and Sigma in the brain structural covariance network of patients with CSM were significantly smaller than those of HCs. These results suggest that the brain structural covariance network of CSM patient exhibits an unoptimizable topological organization with lower $\mathrm{Cp}$ and shorter Lp. In addition, we also observed larger global efficiency and smaller local efficiency in the brain structural covariance network of patients with CSM compared to that of HCs. Global efficiency is inversely related to Lp and measures the capacity of information transfer between nodes by multiple parallel paths (Achard and Bullmore, 2007). Decrease in Lp indicates the enhanced ability for serial information transfer between remote regions of the brain (Rubinov and Sporns, 2010). 
Increase in global efficiency indicates the intensive capacity for parallel information transfer between brain regions via multiple series of paths. Previous studies have demonstrated that the clustering coefficient can be regarded as a measure of the local efficiency of information transfer (Achard and Bullmore, 2007). Decreases in local efficiency and $\mathrm{Cp}$ indicate the inefficient information transfer in the immediate neighborhoods of each node and a weaker local specialization and modular processing in adjacent neighborhoods (Rubinov and Sporns, 2010). Studies have demonstrated that cortical reorganization is an innate attribute of patients with CSM to compensate for motor damage after SCI (Tan et al., 2015; Bhagavatula et al., 2016). Synaptic plasticity involving modification of pre-existing connections and anatomical plasticity involving the development of new circuitry through sprouting of axons and dendrites are the two main adaptive procedures of cortical reorganization in patients with CSM (Dong et al., 2008). A previous study demonstrated that the brain seems to use available pre-existing neural systems by reducing inhibition in the early recovery stage after SCI. However, during the late stage, the brain appears to enhance the original systems or recruit other systems via neural circuit plasticity (Nishimura and Isa, 2009). It is expected that the cortex reorganization for the development of new circuitry may enhance the information communication and function integration for remote brain regions to recovery sensorimotor function and contribute to the shorter path lengths in the brain structural covariance network of patients with CSM. Although, the lower $\mathrm{Cp}$ and shorter Lp in the brain structural covariance network of CSM patients indicate the topological properties deviate from the attractive small-world model compared to HCs. In the subsequent network resilience analysis, the network resilience of patients with CSM was vulnerable compared to that of HCs in the targeted attack. Decrease in local efficiency also reflects the inferior network resilience to the deletion of individual nodes. It is suggested the low network resilience requires intensive capacity for parallel information transfer and communication across remote brain regions to equilibrate for maintaining the basic biological characteristics of the brain structural covariance network of patients with CSM, which seems to response the innate cortex reorganization after SCI in patients with CSM.

\section{Altered Nodal Betweenness in Patients With CSM}

The betweenness of the SPG.L was significantly higher in patients with CSM than in HCs. Recently, a resting-state functional MRI (fMRI) study has reported increased regional homogeneity in the right SPG in patients with CSM (Tan et al., 2015). Another task fMRI study reported enhancement activation in the SPG and inferior parietal lobule following postoperative recovery of function in patients with CSM (Tam et al., 2010). The neuron functional activity alterations in the SPG of patients with CSM were interpreted to integrate and regulate the injury information from the primary sensory cortex, and greater activity coherence was considered to be required in the somatosensory cortex to compensate for the decreased sensory loss (Tan et al., 2015). In addition, a recent study reported GM atrophy in the SPG.L in subacute CSM patients (Chen et al., 2019), which seems to contradict the increase in nodal betweenness in the SPG.L of patients with CSM in our study. We speculated that the inconsistency may result from the different stage of patients with CSM between the former and the present studies. The former study focused on the subacute stage patients with CSM and the current study did not restrict the condition. However, these results indicate the alterations occurring in the SPG.L of patients with CSM. Nodes with high betweenness or degree are often regarded as network hubs (Bullmore and Sporn, 2009). Network hubs and their connections play pivotal roles in information integration and efficient neuronal signal communication in the central nervous system (van den Heuvel and Sporns, 2013). In brain structural covariance networks, edges correspond to axonal or synaptic links that form the biological infrastructure for neuronal signaling and communication (van den Heuvel and Sporns, 2013). The increased betweenness in the SPG.L of patients with CSM in the current study indicates the high fraction of all shortest paths linking the other axonal or synaptic in the network that pass through it (Rubinov and Sporns, 2010; Cheng et al., 2015) and suggests the fast information transfer between it and the other neurons (He et al., 2008). SPG, also called the somatosensory association cortex, is an important part of the somatosensory system by receiving the nearby primary somatosensory cortex fibers (Chen et al., 2019). It is speculated that the increased information flow and fast information communication between the SPG.L and other cortical regions may facilitate the recovery of somatosensory function. However, further study with more empirical data may be helpful to investigate the issue. It is worth noting that we didn't carry out the network hub identification and distribution analysis in the study. Based on the demonstration of the previous study, we treated the SPG.L as the network hub in the network of the patients with CSM. However, an analysis on nodal betweenness-based hub identification and distribution is worth being implemented in the further study to verify the opinion.

In the current study, we also observed increased betweenness in the SMA.L of patients with CSM. The SMA, together with the pre-supplementary motor area, constitutes the medial part of Brodmann area 6C (Nachev et al., 2008). It is located in the dorsomedial frontal cortex, anatomically anterior to the leg representation of the primary motor cortex (Picard and Strick, 1996, 2001). Through retrograde tracing methods, anatomical studies have shown that the pattern of termination of the SMA corticospinal cells resembles that of primary motor cortex projections (He et al., 1995; Wise, 1996). The SMA cells make direct connections to motor neurons and are related to motor output (Dum and Strick, 1991, 1996). A study on post-surgery patients with CSM reported that improvement in dexterity and finer movements of the upper limbs are associated with recruitment areas other than the premotor cortex, the postcentral gyrus and supplementary motor cortices were also recruited to compensate for the damage in the cervical spinal cord (Bhagavatula et al., 2016). Meanwhile, another study reported that patients with CSM exhibit areas of expanded cortical representation, including the adjacent motor territories, SMA, for the affected limb, compared with healthy participants (Holly et al., 2007). A few neurological functions 
are preserved in patients with CSM by modification of preexisting connections and recruitment of new pathways in a process similar to that observed in patients with stroke or cervical myelitis (Holly et al., 2007). At the network level, integrative functions are performed by a specific set of brain regions and their anatomical connections and depend on neural communication of interregional projections in the network (Goldman-Rakic, 1988; Zeki and Shipp, 1988; van den Heuvel and Sporns, 2013). Perfect integrative functions facilitate largescale patterns of synchronization and information flow between connected elements (Singer, 1993; Brovelli et al., 2004). The dynamic performances of the network can be quantified by nodal betweenness and degree ( $\mathrm{He}$ et al., 2008). That is, the higher betweenness or degree of a node, the more effective information transformation between that and other nodes. In our study, the increased betweenness of the SMA.L in patients with CSM is in accordance with the previously reported rearrangement and recruitment of connections between the SMA.L and motor cortex neurons (Holly et al., 2007; Bhagavatula et al., 2016), suggesting the inherent ability of the brain to improve the efficiency of information transfer and to adapt to changes in the environment.

Moreover, we also observed that the betweenness in the MOG.R was significantly lower in patients with CSM than in HCs. MOG is associated with visual information processing. A recent resting-state fMRI study has reported that the amplitude of low-frequency fluctuations and regional homogeneity in the occipital lobe are decreased in patients with CSM (Chen et al., 2018). The two resting-state functional measures indicate functional activity oscillations (Zang et al., 2007) and synchronization of neurons (Zang et al., 2004), respectively. The decreases of the two brain functional metrics observed in patients with CSM indicate inactivity of the neurons in the occipital lobe cortex. In addition, another study observed decreased WM volume in the right occipital lobule and calcarine gyrus in patients with subacute incomplete cervical cord injury (Chen et al., 2019). The alterations of nodal properties in the MOG.R in our study are in line with previous functional and structural findings in the occipital lobe of patients with CSM. For most patients with CSM, cervical vertigo is also a general symptom that often causes gait instability (Yarbrough et al., 2012). The decreased betweenness in the MOG.R may be associated with altered visual input and output of the occipital lobe in patients with CSM.

It is worth noting that both the previous studies and our study have observed changes in the SPG.L of patients with CSM; the current study further reported changes in the SMA.L. Previous studies focusing on the ipsilateral hemisphere of patients with CSM have demonstrated that the asymmetrical spinal cord compression or asymmetrical spinothalamic sensory loss may be the main interpretation for the changes observed in the ipsilateral hemisphere of patients with CSM (Dong et al., 2008; Zhou et al., 2014; Tan et al., 2015). Additionally, the inconsistent maturity of inter-hemispheric regions has been regarded as another explanation for the changes observed in the ipsilateral hemisphere of patients with CSM (Dong et al., 2008). Though, further study with advanced techniques such as diffusion tensor imaging and advanced statistical analysis may benefit the clarification of the issue.

\section{Network Resilience in the Two Groups}

In network resilience analysis, both the groups including the patients with CSM and the HCs showed equal resilience to random failure. When the number of nodes removed in the targeted attack was in the range of 37 to 60 , the maximum relative size of giant connected components was larger in HCs than in patients with CSM by approximately $10 \%$, upon removal of 44 nodes. A previous similar study ( $\mathrm{He}$ et al., 2008) reported similar resilience to random failure in both patients with Alzheimer's disease and HCs. However, when 25\% core nodes were removed in the targeted attack, the relative size of giant connected components of the Alzheimer's disease group was reduced by nearly $20 \%$ compared with that of HCs, implying vulnerability of the former group to targeted attack. In the present study, the maximum difference of the relative size of giant connected components between the two groups was limited to $10 \%$ during the entire targeted attack. Statistically, we observed the network resilience of patients with CSM was significantly vulnerable compared to HCs upon removal of several different number of nodes. However, across the entire removal of nodes in targeted attack, the resilience difference between the two groups in our study was less obvious than that in the previous Alzheimer's disease study. A previous complete thoracic SCI study demonstrated that the remaining motor fibers within the spinal cord are repaired after SCI, but permanent deficits in motor control may still persist (Wrigley et al., 2008). Ongoing degeneration of the transected corticospinal tract may limit the recovery, and changes occurring in fiber tracts above the injured spinal cord level may also hinder the capacity for full motor recovery following SCI (Wrigley et al., 2008). Thus, despite innate cortex reorganization after SCI, patients with CSM may also present a pathological mechanism analogous to that of patients with complete thoracic SCI. However, further research is needed to confirm this assumption. We speculated that the balance and interplay between cortex reorganization and ongoing degeneration may explain the relative mild vulnerability of patients with CSM.

\section{Study Limitations}

Some limitations of the study need to be acknowledged. First, we constructed the brain structural covariance network based on the GMV morphological descriptor. The combined use of cortical thickness may provide novel insights into the complex network properties of patients with CSM. In addition, a whole brain voxel-wise GM analysis provide fundamental GM alteration evidence which should be supportive for the brain structural covariance network analysis. Secondly, the study only discussed the changes of GM. Performing diffusion tensor imaging analyses of WM fiber tracts may provide additional structural information on cortex reorganization in CSM. Thirdly, the non-parametric permutation test for each network measures at each density was not corrected by multiple comparison. Though, the AUC analysis which alleviates the sensitivity of the between group comparison to the thresholding process should improve the validity of the analysis in the study. Moreover, the topological measures only 
displayed significant differences between the two groups at several network densities. The statistic results seems to be not very robust, which maybe related to the choice of the anatomical parcellation scheme. We took the 90 brain region of AAL atlas as the nodes to construct the brain structural covariance network in this study. The Harvard-Oxford atlas maybe also worth being adopted in the further brain network studies, which should contribute to improve the validity of the statistic significance (Desikan et al., 2006). Finally, we only assessed the topological features of patients with CSM without decompression surgery. Decompression surgery in CSM may relieve symptoms and facilitate cortex reorganization and subsequent recovery of the essential neurological function (Fawcett et al., 2007). Further researches including patients with CSM before and after decompression surgery could provide more clues about cortex reorganization in CSM.

\section{CONCLUSION}

Here, we described brain structural covariance network topological properties of patients with CSM. Our main findings were as follows: (1) The observed global network measures alternations in patients with CSM patients reflect that the brain structural covariance network exhibits the less optimal small-world model compared to that in HCs. (2) Increased betweenness in the SPG.L and SMA.L seems to be related to cortex reorganization to recover multiple sensory functions after spinal cord injury in patients with CSM. (3) The network resilience of patients with CSM exhibiting a relatively mild vulnerability compared to that of HCs is probably attributable to the balance and interplay between cortex reorganization

\section{REFERENCES}

Achard, S., and Bullmore, E. (2007). Efficiency and cost of economical brain functional networks. PLoS Comput. Biol. 3:e17. doi: 10.1371/journal.pcbi. 0030017

Achard, S., Salvador, R., Whitcher, B., Suckling, J., and Bullmore, E. (2006). A resilient, low-frequency, small-world human brain functional network with highly connected association cortical hubs. J. Neurosci. 26, 63-72. doi: 10.1523/ JNEUROSCI.3874-05.2006

Alexander-Bloch, A., Giedd, J. N., and Bullmore, E. (2013). Imaging structural co-variance between human brain regions. Nat. Rev. Neurosci. 14, 322-336. doi: $10.1038 / \mathrm{nrn} 3465$

Ashburner, J. (2007). A fast diffeomorphic image registration algorithm. Neuroimage 38, 95-113. doi: 10.1016/j.neuroimage.2007.07.007

Ashburner, J., and Friston, K. J. (2005). Unified segmentation. Neuroimage 26, 839-851. doi: 10.1016/j.neuroimage.2005.02.018

Barthélemy, M. (2004). Betweenness centrality in large complex networks. Eur. Phys. J. B 38, 163-168. doi: 10.1140/epjb/e2004-00111-4

Bhagavatula, I. D., Shukla, D., Sadashiva, N., Saligoudar, P., Prasad, C., and Bhat, D. I. (2016). Functional cortical reorganization in cases of cervical spondylotic myelopathy and changes associated with surgery. Neurosurg. Focus 40:E2. doi: 10.3171/2016.3.FOCUS1635

Brovelli, A., Ding, M., Ledberg, A., Chen, Y., Nakamura, R., and Bressler, S. L. (2004). Beta oscillations in a large-scale sensorimotor cortical network: directional influences revealed by Granger causality. Proc. Natl. Acad. Sci. U.S.A. 101, 9849-9854. doi: 10.1073/pnas.0308538101 and ongoing degeneration. These findings contribute to the understanding of cortex reorganization in CSM from a structural network viewpoint.

\section{DATA AVAILABILITY STATEMENT}

The datasets presented in this article are not readily available because to protect the subjects' privacy. Requests to access the datasets should be directed to YZ, fskyanteam@126.com.

\section{ETHICS STATEMENT}

The studies involving human participants were reviewed and approved by Ethics Committee for Clinical Research of Renmin Hospital of Wuhan University. The patients/participants provided their written informed consent to participate in this study.

\section{AUTHOR CONTRIBUTIONS}

CK and YZ designed the study, collected and analyzed the data, and interpreted the results of the analysis. CK wrote the manuscript. CL and JC contributed to the discussion and manuscript revision. All authors listed meet the criteria for authorship, read, and approved the final manuscript.

\section{ACKNOWLEDGMENTS}

We thank all the participants for their time and effort.

Bullmore, E., and Sporn, O. (2009). Complex brain networks: graph theoretical analysis of structural and functional systems. Nat. Rev. Neurosci. 10, 186-198. doi: $10.1038 / \mathrm{nrn} 2575$

Bullmore, E. T., Suckling, J., Overmever, S., Rabe-Hesketh, S., Taylor, E., and Brammer, M. J. (1999). Global, voxel and cluster tests, by theory and permutation, for a difference between two groups of structural MR images of the brain. IEEE Trans. Med. Imaging 18, 32-42. doi: 10.1109/42.750253

Chen, Q., Zheng, W., Chen, X., Li, X., Wang, L., Qin, W., et al. (2019). Reorganization of the somatosensory pathway after subacute incomplete cervical cord injury. Neuroimage Clin. 21:101674. doi: 10.1016/j.nicl.2019. 101674

Chen, Z., Wang, Q., Liang, M., Zhao, R., Zhu, J., Xiong, W., et al. (2018). Visual cortex neural activity alteration in cervical spondylotic myelopathy patients: a resting-state fMRI study. Neuroradiology 60, 921-932. doi: 10.1007/s00234018-2061-x

Cheng, H., Newman, S., Goni, J., Kent, J. S., Howell, J., Bolbecker, A., et al. (2015). Nodal centrality of functional network in the differentiation of schizophrenia. Schizophr. Res. 168, 345-352. doi: 10.1016/j.schres.2015.08.011

Desikan, R. S., Segonne, F., Fischl, B., Quinn, B. T., Dickerson, B. C., Blacker, D., et al. (2006). An automated labeling system for subdividing the human cerebral cortex on MR scans into gyral based regions of interest. Neuroimage 31, 968-980. doi: 10.1016/j.neuroimage.2006.01.021

Dong, Y., Holly, L. T., Albistegui-Dubois, R., Yan, X., Marehbian, J., Newton, J. M., et al. (2008). Compensatory cerebral adaptations before and evolving changes after surgical decompression in cervical spondylotic myelopathy. J. Neurosurg. Spine 9, 538-551. doi: 10.3171/SPI.2008.10.0831 
Duggal, N., Rabin, D., Bartha, R., Barry, R. L., Gati, J. S., Kowalczyk, I., et al. (2010). Brain reorganization in patients with spinal cord compression evaluated using fMRI. Neurology 74, 1048-1054. doi: 10.1212/WNL.0b013e3181d6b0ea

Dum, R. P., and Strick, P. L. (1991). The origin of corticospinal projections from the premotor areas in the frontal lobe. J. Neurosci. 11, 667-689. doi: 10.1523/ JNEUROSCI.11-03-00667.1991

Dum, R. P., and Strick, P. L. (1996). Spinal cord terminations of the medial wall motor areas in macaque monkeys. J. Neurosci. 16, 6513-6525. doi: 10.1523/ JNEUROSCI.16-20-06513.1996

Evans, A. C. (2013). Networks of anatomical covariance. Neuroimage 80, 489-504. doi: 10.1016/j.neuroimage.2013.05.054

Fawcett, J. W., Curt, A., Steeves, J. D., Coleman, W. P., Tuszynski, M. H., Lammertse, D., et al. (2007). Guidelines for the conduct of clinical trials for spinal cord injury as developed by the ICCP panel: spontaneous recovery after spinal cord injury and statistical power needed for therapeutic clinical trials. Spinal Cord 45, 190-205. doi: 10.1038/sj.sc.3102007

Freeman, L. C. (1977). A set of measures of centrality based on betweenness. Sociometry 40, 35-41. doi: 10.2307/3033543

Goldman-Rakic, P. S. (1988). Topography of cognition: parallel distributed networks in primate association cortex. Annu. Rev. Neurosci. 11, 137-156. doi: 10.1146/annurev.ne.11.030188.001033

Grabher, P., Callaghan, M. F., Ashburner, J., Weiskopf, N., Thompson, A. J., Curt, A., et al. (2015). Tracking sensory system atrophy and outcome prediction in spinal cord injury. Ann. Neurol. 78, 751-761. doi: 10.1002/ana.24508

He, S.-Q., Dum, R. P., and Strick, P. L. (1995). Topographic organization of corticospinal projections from the frontal lobe: motor areas on the medial surface of the hemisphere. J. Neurosci. 15, 3284-3306. doi: 10.1111/j.2042-3292. 1996.tb01657.x

He, Y., Chen, Z., and Evans, A. (2007). Small-world anatomical networks in the human brain revealed by cortical thickness from MRI. Cereb. Cortex 17, 2407-2419. doi: 10.1093/cercor/bhl149

He, Y., Chen, Z., and Evans, A. (2008). Structural insights into aberrant topological patterns of large-scale cortical networks in Alzheimer's disease. J. Neurosci. 28, 4756-4766. doi: 10.1523/JNEUOSCI.0141-08.2008

He, Y., Dagher, A., Chen, Z., Charil, A., Zijdenbos, A., Worsley, K., et al. (2009). Impaired small-world efficiency in structural cortical networks in multiple sclerosis associated with white matter lesion load. Brain 132, 3366-3379. doi: 10.1093/brain/awp089

Holly, L. T. (2009). Management of cervical spondylotic myelopathy with insights from metabolic imaging of the spinal cord and brain. Curr. Opin. Neurol. 22, 575-581. doi: 10.1097/WCO.0b013e3283325ea7

Holly, L. T., Dong, Y., Albistegui-Dubois, R., Marehbian, J., and Dobkin, B. (2007). Cortical reorganization in patients with cervical spondylotic myelopathy. J. Neurosurg. Spine 6, 544-551. doi: 10.3171/spi.2007.6.6.5

Hosseini, S. M., Hoeft, F., and Kesler, S. R. (2012). GAT: a graph-theoretical analysis toolbox for analyzing between-group differences in large-scale structural and functional brain networks. PLoS One 7:e40709. doi: 10.1371/journal.pone. 0040709

Hou, J. M., Yan, R. B., Xiang, Z. M., Zhang, K., Liu, J., Wu, Y. T., et al. (2014). Brain sensorimotor system atrophy during the early stage of spinal cord injury in humans. Neuroscience 266, 208-215. doi: 10.1016/j.neuroscience.2014. 02.013

Jutzeler, C. R., Huber, E., Callaghan, M. F., Luechinger, R., Curt, A., Kramer, J. L., et al. (2016). Association of pain and CNS structural changes after spinal cord injury. Sci. Rep. 6:18534. doi: 10.1038/srep18534

Kaiser, M., and Hilgetag, C. C. (2006). Nonoptimal component placement, but short processing paths, due to long-distance projections in neural systems. PLoS Comput. Biol. 2:e95. doi: 10.1371/journal.pcbi.0020095

Kaiser, M., Martin, R., Andras, P., and Young, M. P. (2007). Simulation of robustness against lesions of cortical networks. Eur. J. Neurosci. 25, 3185-3192. doi: 10.1111/j.1460-9568.2007.05574.x

Kowalczyk, I., Duggal, N., and Bartha, R. (2012). Proton magnetic resonance spectroscopy of the motor cortex in cervical myelopathy. Brain 135, 461-468. doi: 10.1093/brain/awr328

Liu, F., Tian, H., Li, J., Li, S., and Zhuo, C. (2019). Altered voxel-wise gray matter structural brain networks in schizophrenia: association with brain genetic expression pattern. Brain Imaging Behav. 13, 493-502. doi: 10.1007/s11682018-9880-6
Liu, F., Zhou, C., and Yu, C. (2016). Altered cerebral blood flow covariance network in schizophrenia. Front. Neurosci. 10:308. doi: 10.3389/fnins.2016.00308

Lv, B., Li, J., He, H., Li, M., Zhao, M., Ai, L., et al. (2010). Gender consistency and difference in healthy adults revealed by cortical thickness. Neuroimage 53, 373-382. doi: 10.1016/j.neuroimage.2010.05.020

Nachev, P., Kennard, C., and Husain, M. (2008). Functional role of the supplementary and pre-supplementary motor areas. Nat. Rev. Neurosci. 9, 856-869. doi: 10.1038/nrn2478

Nishimura, Y., and Isa, T. (2009). Compensatory changes at the cerebral cortical level after spinal cord injury. Neuroscientist 15, 436-444. doi: 10.1177/ 1073858408331375

Picard, N., and Strick, P. L. (1996). Motor areas of the medial wall: a review of their location and functional activation. Cereb. Cortex 6, 342-353. doi: 10.1093/ cercor/6.3.342

Picard, N., and Strick, P. L. (2001). Imaging the premotor areas. Curr. Opin. Neurobiol. 11, 663-672. doi: 10.1016/S0959-4388(01)00266-5

Rubinov, M., and Sporns, O. (2010). Complex network measures of brain connectivity: uses and interpretations. Neuroimage 52, 1059-1069. doi: 10.1016/ J.NEUROIMAGE.2009.10.003

Sharp, N. J. H., and Simon, J. W. (2005). Cervical Disc Disease-Small Animal Spinal Disorders, 2nd Edn. Amsterdam: Elsevier, 93-120. doi: 10.1016/B978-0-72343209-8.50011-X

Shi, F., Yap, P. T., Wei, G., Lin, W., Gilmore, J. H., and Shen, D. (2012). Altered structural connectivity in neonates at genetic risk for schizophrenia: a combined study using morphological and white matter networks. Neuroimage 62, 16221633. doi: 10.1016/j.neuroimage.2012.05.026

Singer, W. (1993). Synchronization of cortical activity and its putative role in information processing and learning. Annu. Rev. Physiol. 55, 349-374. doi: 10.1146/annurev.ph.55.030193.002025

Sone, D., Watanabe, M., Maikusa, N., Sato, N., Kimura, Y., and Enokizono, M. (2019). Reduced resilience of brain gray matter networks in idiopathic generalized epilepsy: a graph-theoretical analysis. PLoS One 14:e0212494. doi: 10.1371/journal.pone.0212494

Sporns, O., Tononi, G., and Kotter, R. (2005). The human connectome: a structural description of the human brain. PLoS Comput. Biol. 1:e42. doi: 10.1371/journal. pcbi.0010042

Tam, S., Barry, R. L., Bartha, R., and Duggal, N. (2010). Changes in functional magnetic resonance imaging cortical activation after decompression of cervical spondylosis: case report. Neurosurgery 67, E863-E864. doi: 10.1227/01.NEU. 0000374848.86299.17

Tan, Y., Zhou, F., Wu, L., Liu, Z., Zeng, X., Gong, H., et al. (2015). Alteration of regional homogeneity within the sensorimotor network after spinal cord decompression in cervical spondylotic myelopathy: a resting-state fMRI study. Biomed Res. Int. 2015:647958. doi: 10.1155/2015/647958

Tao, G., Guan, X., Zeng, Q., Xuan, M., Gu, Q., Huang, P., et al. (2018). Alterations of brain structural network in Parkinson's disease with and without rapid eye movement sleep behavior disorder. Front. Neurol. 9:334. doi: 10.3389/fneur. 2018.00334

Tzourio-Mazoyer, N., Landeau, B., Papathanassiou, D., Crivello, F., Etard, O., Delcroix, N., et al. (2002). Automated anatomical labeling of activations in SPM using a macroscopic anatomical parcellation of the MNI MRI single-subject brain. Neuroimage 15, 273-289. doi: 10.1006/nimg.2001.0978

van den Heuvel, M. P., and Sporns, O. (2013). Network hubs in the human brain. Trend Cogn. Sci. 17, 683-696. doi: 10.1016/j.tics.2013.09.012

Watts, D. J., and Strogatz, S. H. (1998). Collective dynamics of small world networks. Nature 393, 440-442. doi: 10.1038/30918

Wen, W., He, Y., and Sachdev, P. (2011). Structural brain networks and neuropsychiatric disorders. Curr. Opin. Psychiatry 24, 219-225. doi: 10.1097/ YCO.0b013e32834591f8

Wise, S. P. (1996). Corticospinal efferents of the supplementary sensorimotor area in relation to the primary motor area. Adv. Neurol. 70, 57-69.

Wrigley, P. J., Gustin, S. M., Macey, P. M., Nash, P. G., Gandevia, S. C., Macefield, V. G., et al. (2008). Anatomical changes in human motor cortex and motor pathways following complete thoracic spinal cord injury. Cereb. Cortex 19, 224-232. doi: 10.1093/cercor/bhn072

Yao, Z., Zhang, Y., Lin, L., Zhou, Y., Xu, C., and Jiang, T. (2010). Abnormal cortical networks in mild cognitive impairment and Alzheimer's disease. PLoS Comput. Biol. 6:e1001006. doi: 10.1371/journal.pcbi.1001006 
Yarbrough, C. K., Murphy, R. K., Ray, W. Z., and Stewart, T. J. (2012). The natural history and clinical presentation of cervical spondylotic myelopathy. Adv. Orthop. 2012:480643. doi: 10.1155/2012/480643

Yonenobu, K., Abumi, K., Nagata, K., Taketomi, E., and Ueyama, K. (2001). Interobserver and intraobserver reliability of the Japanese orthopaedic association scoring system for evaluation of cervical compression myelopathy. Spine 26, 1890-1894. doi: 10.1097/00007632-20010901000014

Zang, Y., Jiang, T., Lu, Y., He, Y., and Tian, L. (2004). Regional homogeneity approach to fMRI data analysis. Neuroimage 22, 394-400. doi: 10.1016/j. neuroimage.2003.12.030

Zang, Y. F., He, Y., Zhu, C., Cao, Q., Sui, M., Liang, M., et al. (2007). Altered baseline brain activity in children with ADHD revealed by restingstate functional MRI. Brain Dev. 29, 83-91. doi: 10.1016/j/braindev.2006. 07.002

Zeki, S., and Shipp, S. (1988). The functional logic of cortical connections. Nature 335, 311-317. doi: 10.1038/335311a0

Zhou, F., Gong, H., Liu, X., Wu, L., Luk, K., and Hu, Y. (2014). Increased lowfrequency oscillation amplitude of sensorimotor cortex associated with the severity of structural impairment in cervical myelopathy. PLoS One 9:e104442 doi: 10.1371/journal.pone.0104442

Zou, T., She, L., Zhan, C., Gao, Y., and Chen, H. (2018). Altered topological properties of gray matter structural covariance networks in minimal hepatic encephalopathy. Front. Neuroanat. 12:101. doi: 10.3389/fnana.2018.00101

Conflict of Interest: The authors declare that the research was conducted in the absence of any commercial or financial relationships that could be construed as a potential conflict of interest.

The reviewer LG declared a shared affiliation, though no other collaboration, with the authors to the handling Editor.

Copyright (c) 2020 Kuang, Zha, Liu and Chen. This is an open-access article distributed under the terms of the Creative Commons Attribution License (CC BY). The use, distribution or reproduction in other forums is permitted, provided the original author(s) and the copyright owner(s) are credited and that the original publication in this journal is cited, in accordance with accepted academic practice. No use, distribution or reproduction is permitted which does not comply with these terms. 